\title{
Utility of a modified components separation for abdominal wall reconstruction in the liver and kidney transplant population
}

\author{
Cara K Black ${ }^{1}$, Elizabeth G Zolper ${ }^{1}$, Elliot T Walters ${ }^{2}$, Jessica Wang ${ }^{2}$, Jesus Martinez ${ }^{1}$, \\ Andrew Tran ${ }^{1}$, Iram Naz ${ }^{2}$, Vikas Kotha ${ }^{2}$, Paul J Kim², Sarah R Sher ${ }^{2}$, Karen K Evans ${ }^{2}$ \\ ${ }^{1}$ Georgetown University School of Medicine, Washington, DC; ${ }^{2}$ Department of Plastic and Reconstructive Surgery, MedStar Georgetown \\ University Hospital, Washington, DC, USA
}

Background Incisional hernia is a common complication following visceral organ transplantation. Transplant patients are at increased risk of primary and recurrent hernias due to chronic immune suppression and large incisions. We conducted a retrospective review of patients with a history of liver or kidney transplantation who underwent hernia repair to analyze outcomes and hernia recurrence.

Methods This is a single center, retrospective review of 19 patients who received kidney and/or liver transplantation prior to presenting with an incisional hernia from 2011 to 2017 . All hernias were repaired with open component separation technique (CST) with biologic mesh underlay.

Results The mean age of patients was $61.0 \pm 8.3$ years old, with a mean body mass index of $28.4 \pm 4.8 \mathrm{~kg} / \mathrm{m}^{2}, 15$ males (78.9\%), and four females (21.1\%). There were seven kidney, 11 liver, and one combined liver and kidney transplant patients. The most common comorbidities were hypertension (16 patients, 84.2\%), diabetes (9 patients, 47.4\%), and tobacco use (8 patients, 42.1\%). Complications occurred in six patients (31.6\%) including hematoma (1/19), abscess (1/19), seroma (2/19), and hernia recurrence (3/19) at mean follow-up of $28.7 \pm 22.8$ months. With the exception of two patients with incomplete follow-up, all patients healed at a median time of 27 days.

Conclusions This small, retrospective series of complex open CST in transplant patients shows acceptable rates of long-term hernia recurrence and healing. By using a multidisciplinary approach for abdominal wall reconstruction, we believe that modified open CST with biologic mesh is a safe and effective technique in the transplant population with complex abdominal hernias.

Keywords Transplants / Surgical mesh / Incisional hernia / Immunosuppression / Abdominal wall

Received: November 15, 2018 - Revised: July 13, $2019 \bullet$ Accepted: September 1, 2019

pISSN: 2234-6163 • elSSN: 2234-6171 • https://doi.org/10.5999/aps.2018.01361 • Arch Plast Surg 2019;46:462-469
Correspondence: Karen K Evans Department of Plastic and Reconstructive Surgery, MedStar Georgetown University Hospital, 3800 Reservoir Rd NW, Washington, DC 20007, USA

Tel: +1-202-444-8751

Fax: +1-202-444-0300

E-mail:

Karen.K.Evans@gunet.georgetown.edu

This article was presented at the American Society of Plastic Surgery, on September 28-October 1, 2018, in Chicago, IL, USA.

We would like to acknowledge Eshetu Tefera, the department's biostatistician, for his help with the statistics. We would also like to thank Marielle Mahan, a Georgetown University medical student, for helping with the medical illustrations.

\section{INTRODUCTION}

Incisional hernia $(\mathrm{IH})$ is a common complication of solid organ transplantation (SOT) with significant consequences to patient quality of life. The incidence of IH in the SOT population has been reported to be $7.5 \%$ overall [1]. However, the incidence 
widely varies specific to organ of transplantation. The incidence of $\mathrm{IH}$ after renal transplant is reported to be $3.6 \%$ to $7.04 \%$ in comparison to $4.6 \%$ to $23 \%$ after hepatic transplant [1-4]. Predisposing factors for $\mathrm{IH}$ after SOT include: obesity, cirrhosis, smoking history, immunosuppression, duration of procedure, repeat operations, concurrent abdominal wall hernia, development of fluid collections, and surgical site infection (SSI) [1,5-8].

IH repair, a procedure considered routine in an otherwise healthy patient, is significantly more complex in the transplant population. Patients often have a history of multiple abdominal operations including laparotomies and prior hernia repairs. Additionally, transplant patients commonly have large non-midline incisions such as Mercedes, Gibson, or subcostal incisions utilized for SOT. These factors can result in significant distortion of the native tissue planes thus complicating subsequent $\mathrm{IH}$ repair. The standard open component separation technique (CST) must be modified in this patient population and an understanding of the anatomic layers that are involved in such hernias must be understood in order to properly repair the defects. In the Chevron IH defect there is both a vertical and horizontal component that must be repaired. In addition, the rectus muscles may be de-innervated. In the Gibson IH defect, the defect is usually located at the edge of the rectus muscle and the linea semilunaris. The CST in these defects requires a posterior external oblique (EO) dissection and sometimes requires a lateral division of the muscle itself in order to bring it back to the edge of rectus. It is critical to anchor the mesh to the inguinal ligament in the caudal aspect of the hernia.

Various methods of IH repair have been used in the transplant population such as synthetic mesh, biological mesh, component separation, or autologous free tissue transfer including: tensor fascia lata grafts, flaps from the thighs, and combinations of these procedures [8]. The use of synthetic mesh carries increased risk in the immunosuppressed transplant population including higher rates of SSI [6,9-11]. Furthermore, placement of mesh can complicate future operative approaches specifically by limiting access to the graft for revision, retransplantation, or explantation.

The following study is a retrospective case series describing the outcomes of complex abdominal wall repair in the setting of solid organ transplant patients with chronic immunosuppression. The goal of this study was to review the multidisciplinary experience at our institution with CST combined with biologic mesh in the immunosuppressed transplant population. The aim of this analysis was to further the understanding of outcomes such as hernia recurrence and complications in this high-risk population. In addition, we deemed it important to analyze our multidisciplinary approach with transplant surgery and plastic surgery to handling complex abdominal wall reconstruction.

\section{METHODS}

\section{Retrospective review}

Institutional Review Board approval was obtained (IRB No. 2018-0008). A single-center, retrospective review of 19 SOT patients who underwent IH repair at our institution from 2011 to 2017 was conducted. Written informed consents were obtained. All patients received kidney and/or liver transplantation prior to presenting with an IH. All hernias were repaired with open component separation with biologic mesh underlay technique. IH repairs were performed by the plastic and reconstructive surgery service in conjunction with the transplant surgery service. Primary outcomes assessed were wound healing, time to healing, and hernia-related complications including recurrence, seroma, hematoma, abscess, and dehiscence. Pre and postoperative factors were also identified including patient demographics and comorbidities, size of initial defect, closure type, and immunosuppressive regimen.

\section{Surgical technique}

Open, perforator sparing component separation was performed on 19 patients. Transplant surgery performed the laparotomy and lysis of adhesions. Plastic surgery performed the CST, mesh underlay and fascia approximation. In addition, plastic surgery excised soft tissue scar tissue and closed the skin. Written informed consent was obtained from patients for the publication of the accompanying preoperative, intraoperative, and postoperative photographs.

For liver transplant/chevron incisions (Figs. 1, 2), the EO re-

\section{Fig. 1. Incisional hernia following liver transplant}

Preoperative view of incisional hernia following liver transplant. The Chevron hernia is usually in the $\mathrm{T}$ junction of the chevron incision. 
lease was performed as cranial as possible, above the ribs to allow for the upper one-third approximation of the rectus abdominis muscles. The CST for liver transplant incisions did not always require distal one-third release of the $\mathrm{EO}$ since the hernias were located more commonly in the $\mathrm{T}$ junction of the chevron incision (Fig. 1). Biologic mesh (Strattice) was placed in the underlay position, because the in-lay plane in these patients is usually not preserved due to multiple previous laparotomies. The mesh was cut to fit the Chevron, $\mathrm{T}$ IH that contained both a horizontal and vertical component (Fig. 2). The mesh was sutured using 0.0 PDS with appropriate tension. Fascial approximation was achieved in all patients (Fig. 3).

For kidney/Gibson IH repair, the EO was dissected from the internal oblique along the lateral edge of the hernia (Figs. 4, 5).

\section{Fig. 2. Biologic mesh for repair of incisional hernia \\ (A) Biologic mesh for repair of incisional hernia following liver transplant. The mesh was cut to a shape to accommodate both the vertical and horizontal component of the hernia vector. (B) Intra- operative view of cutting the mesh to the shape of the hernia.}
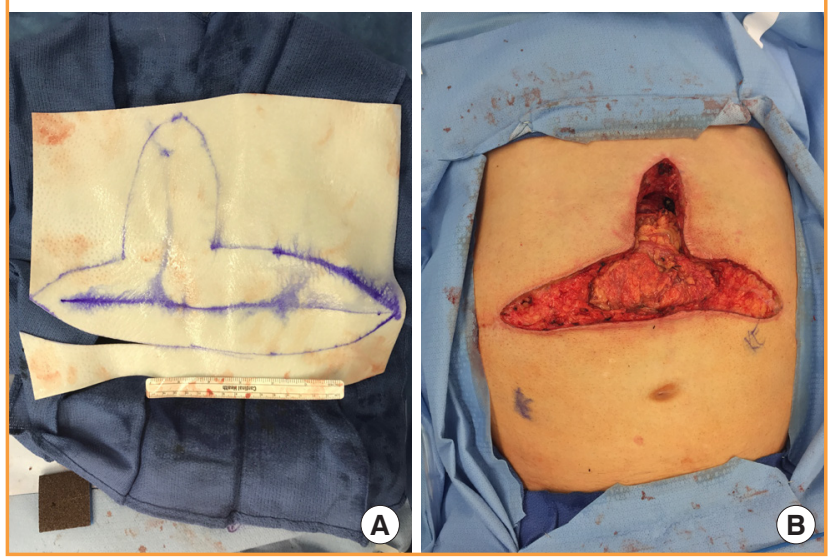

A contralateral EO release was performed if needed to allow for rectus muscle approximation. In addition, a posterior rectus muscle release was performed as needed if the rectus complex was scarred. If needed, the most lateral aspect of the EO muscle could be divided to allow for medialization as long as the blood supply to the muscle is spared. Mesh must be anchored in all quadrants, including caudally along the inguinal ligament (Fig. 5). If the defect was large, the mesh was also anchored into the anterior superior iliac spine and the pubic tubercle.

\section{Statistical analysis}

We calculated descriptive statistics for the outcomes of interest. All statistical analyses were performed using SAS version 9.4 (SAS Institute Inc., Cary, NC, USA). Descriptive statistics were used to describe study subjects. Continuous variables were described by means, standard deviations and range (minimummaximum). Categorical variables were described by frequencies and percentages.

\section{RESULTS}

Over a 6-year period (2011 to 2017), 19 complex IH repairs that combined biologic mesh underlay with complete fascial approximation and CST were performed at a single center in kidney and/or liver transplant patients. Table 1 summarizes the demographics and characteristics of the patients who had the procedure. The mean age of the patients was $61.0 \pm 8.3$ years old (range, 46.4-75.2 years). The mean body mass index was $28.4 \pm 4.8 \mathrm{~kg} / \mathrm{m}^{2}$ (range, $20.1-37.0 \mathrm{~kg} / \mathrm{m}^{2}$ ). The patient population consisted of a higher number of males (15 males), as compared to four females. Comorbidities are shown in Table 1. Hy-

\section{Fig. 3. Mesh underlay placement for incisional hernia repair}

Mesh underlay placement for repair of incisional hernia following liver transplant. After component separation, biologic mesh was applied under the fascia with appropriate tension (A). The tension was distributed unto the mesh which then allowed for primary fascia approximation (B).
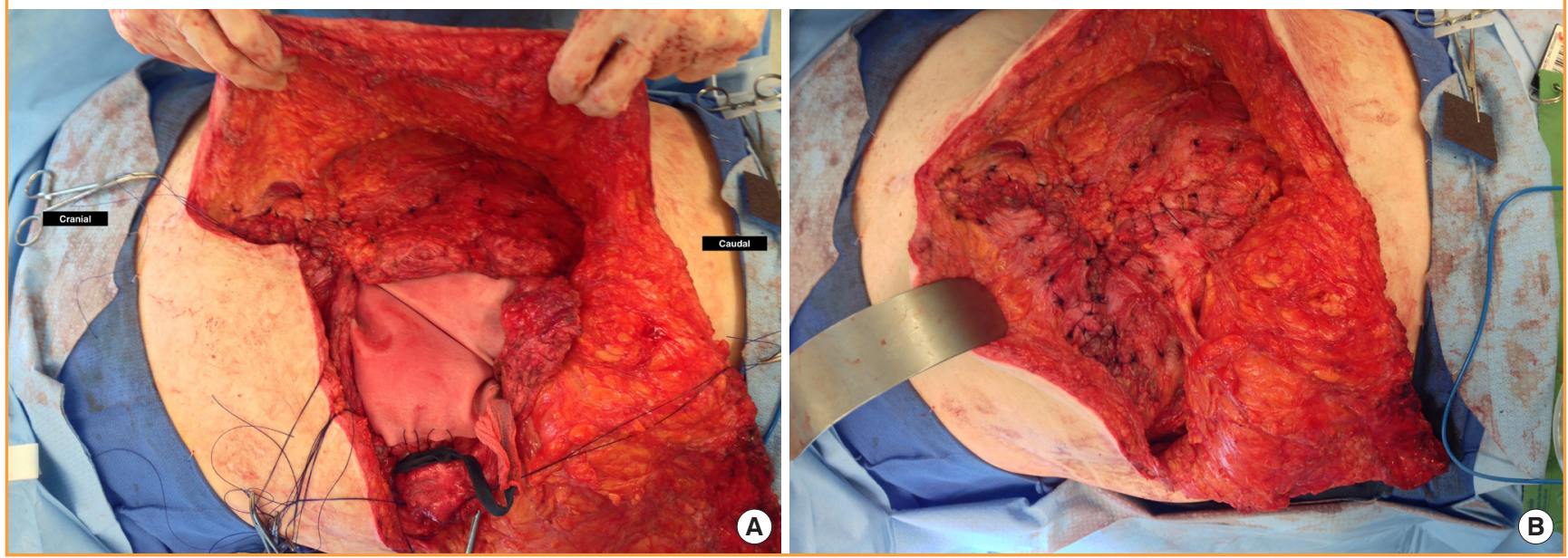
pertension was the most common co-morbidity (16 patients, $84.2 \%)$, followed by diabetes ( 9 patients, $47.4 \%$ ), and history of tobacco use ( 8 patients, $42.1 \%)$.
Mean prior surgeries were $1.0 \pm 1.3$ (range, $0-4.0$ ) per patient, including 10 patients with prior IH repairs. The mean time from original transplant procedure to hernia repair was $31.6 \pm 26.4$

\section{Fig. 4. Modified component separation for incisional hernia repair}

Modified components separation for repair of incisional hernia following kidney transplantation. (A) Preoperative image of a patient with large hernia following kidney transplantation using a Gibson incision. $(B, C)$ Mesh placement underlay and fascial approximation. (D, E) Soft tissue closure included excision of redundant skin. (F) Postoperative images showing hernia repair and healed incision.
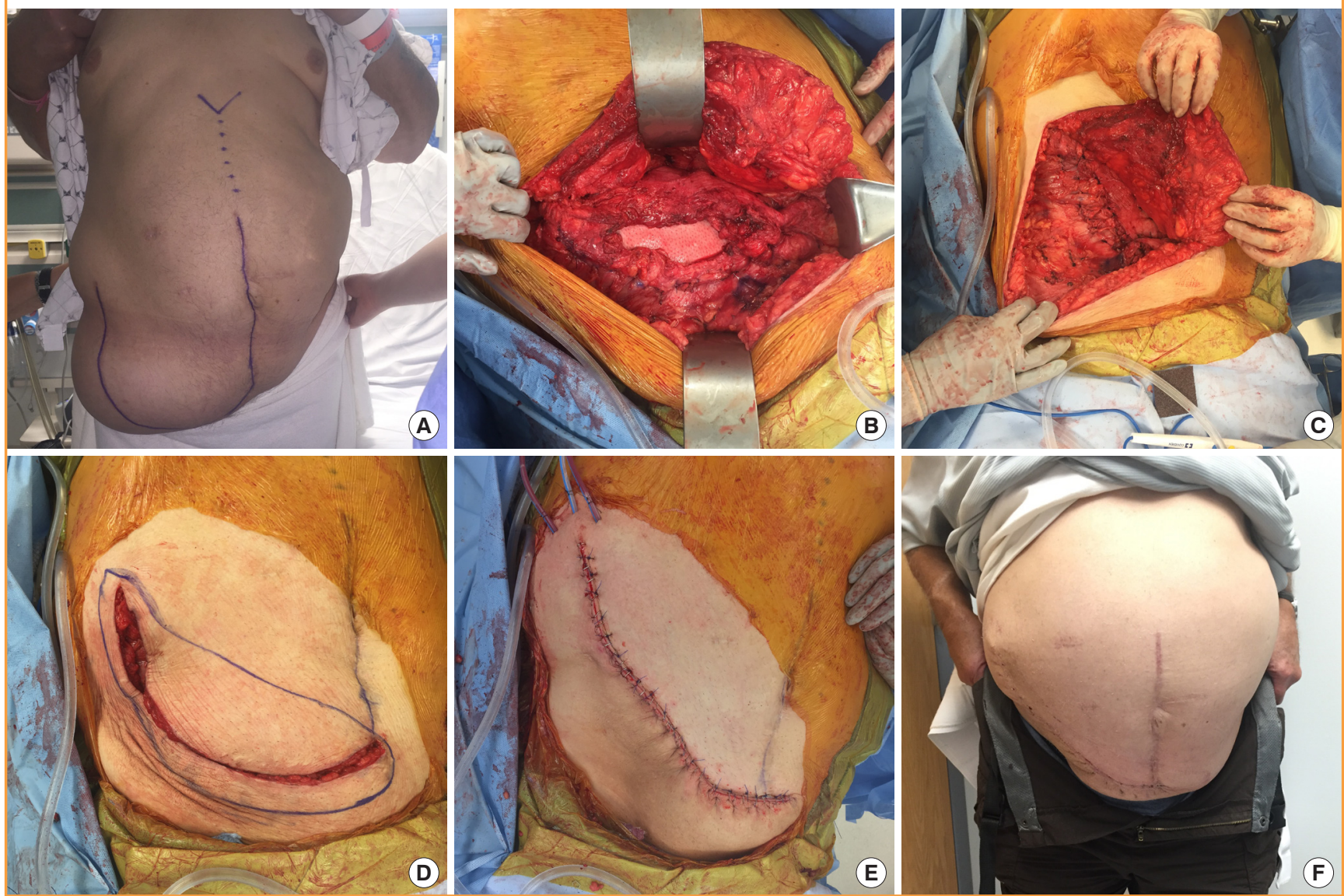

\section{Fig. 5. Illustration of modified CST}

(A) Typical Gibson incisional hernia defect located at the lateral edge of the rectus muscle and the linea semilunaris. (B) Biologic mesh (arrow) is placed in the underlay position and is anchored in all four quadrants including to the inguinal ligament caudally. Dashed line represents extent of external oblique dissection. (C) External oblique is dissected free from internal oblique at lateral edge of defect. (D) External oblique is medialized to approximate with intact rectus muscle and achieve primary fascial repair.

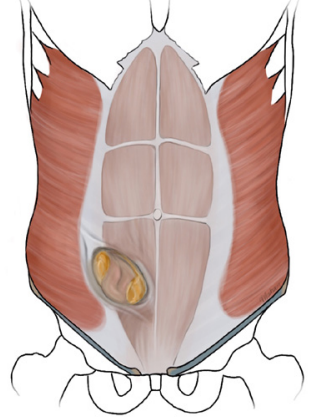

(A)

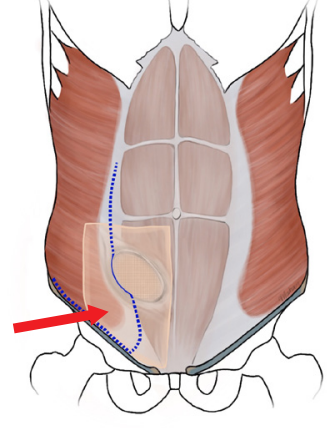

B

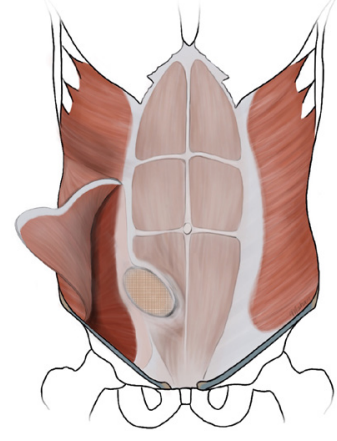

C

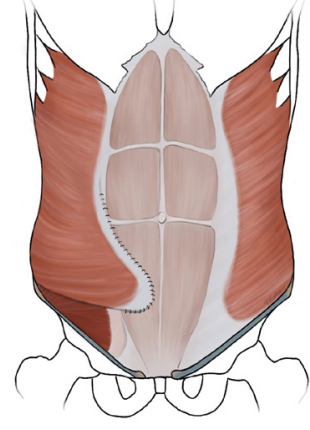

(D) 


\section{Table 1. Patient demographics $(n=19)$}

\begin{tabular}{|lc|}
\hline Variable & Value \\
\hline Age (yr) & $61.0 \pm 8.3(46.4-75.2)$ \\
BMI (kg/m²) & $28.4 \pm 4.8(20.1-37.0)$ \\
Sex & \\
Female & $4(21.1)$ \\
Male & $15(78.9)$ \\
Comorbidities & \\
DM & $9(47.4)$ \\
HTN & $16(84.2)$ \\
CAD & $2(10.5)$ \\
CHF & $2(10.5)$ \\
HLD & $6(31.6)$ \\
PAD & $2(10.5)$ \\
ESRD & $5(26.3)$ \\
Tobacco use & $8(42.1)$ \\
Prior procedures & \\
Prior abdominal surgeries & $2.8 \pm 1.9(1.0-7.0)$ \\
Prior VHR & $10(52.6)$ \\
& $1.0 \pm 1.3(0-4.0)$ \\
Time from SOT to VHR (mon) & $31.6 \pm 26.4(6.0-108.0)$ \\
Transplant type & $7(36.8)$ \\
Kidney & $11(57.9)$ \\
Liver & $1(5.3)$ \\
Liver/kidney & \\
Incisional hernia characteristics & \\
Initial defect size (cm²) & \\
\hline Values are presented as mean \pm SD (range) or number (\%). & \\
BMI, body mass index; DM, diabetes mellitus; HTN, hypertension; CAD, coronary \\
artery disease; CHF, congestive heart failure; HLD, hyperlipidemia; PAD, \\
peripheral artery disease; ESRD, end stage renal disease; VHR, ventral hernia \\
repair; SOT, solid organ transplant. \\
\end{tabular}

months (range, 6.0-108.0 months). There were seven kidney transplant patients, 11 liver transplant patients and one combined liver kidney transplant patient. All patients were on at least one form of immunosuppression (mean, $1.5 \pm 0.5$; range, $1-2)$. However, no patients were on steroids at the time of repair. The average initial defect size was $349.9 \pm 211.4 \mathrm{~cm}^{2}$ (range, $60.0-900.0 \mathrm{~cm}^{2}$ ).

All IHs were repaired with CST and underlay biological mesh (i.e., Strattice) (Table 2). Postoperatively, negative pressure wound therapy (incisional VAC therapy) was used in 12 patients (63.2\%). Complications were experienced by six patients (31.6\%). This included hematoma $(\mathrm{n}=1,5.3 \%)$, abscess $(\mathrm{n}=1$, $5.3 \%)$, seroma $(n=2,10.5 \%)$ (Table 3$)$. Table 4 summarizes hernia recurrence experienced by three out of 19 patients (15.8\%) over an average of $28.7 \pm 22.8$ months (range, 88-52.8 months). One patient (5.3\%) developed an infection (abscess) in the subcutaneous tissue as detected by computed tomography scan and cultures grew Staphylococcus aureus. The abscess was subsequently drained and the mesh did not need to be removed. Of the nine patients who had diabetes, five had complications (55.6\%). The breakdown of complications of each of these five
Table 2. Operative technique and devices

\begin{tabular}{|ll|}
\hline Variable & No. (\%) \\
\hline Biologic mesha) & $19(100.0)$ \\
Porcine dermal mesh & 17 \\
$\quad$ Strattice & 1 \\
Permacol & 1 \\
$\quad$ Unspecified & $19(100.0)$ \\
Component separation & $12(63.2)$ \\
NPWT/dressing & \\
\hline NPWT, negative pressure wound therapy. & \\
a)All of the biologic mesh used was porcine dermal mesh. One was Permacol \\
(cross linked), 17 were Strattice (non-cross linked), and one was unspecified.
\end{tabular}

\section{Table 3. Complications}

\begin{tabular}{|c|c|}
\hline Variable & Value \\
\hline No. of patients with complications & $6(31.6)^{a)}$ \\
\hline Hernia recurrence & $3(15.8)$ \\
\hline Primary VHR & $1(10.0)$ \\
\hline Recurrent VHR & $2(22.2)$ \\
\hline Time to recurrence (mon) & $28.7 \pm 22.8(8-52.8)$ \\
\hline Seroma & $2(10.5)$ \\
\hline Hematoma & $1(5.3)$ \\
\hline Abscess & $1(5.3)$ \\
\hline No. of diabetic patients $(n=9)$ with complications & $5(55.6)$ \\
\hline \multicolumn{2}{|l|}{ Healing and related complications } \\
\hline Healed & $17(89.5)$ \\
\hline Time to skin healing (day) & $27(20-579)^{b}$ \\
\hline Lost to follow-up & $2(10.5)$ \\
\hline Dehiscence & $1(5.3)$ \\
\hline Revision required ${ }^{(c)}$ & $2(10.5)$ \\
\hline \multicolumn{2}{|c|}{$\begin{array}{l}\text { Values are presented as number (\%), mean } \pm \text { SD (range), or median (range). } \\
\text { VHR, ventral hernia repair. } \\
\text { a) One patient had two complications, seroma and hematoma; b)Median healing } \\
\text { time presented. Mean healing time was inaccurate because one patient was lost } \\
\text { to follow-up until } 569 \text { postoperative days. The patient's incisional hernia repair } \\
\text { site was found to be healed at that time; 'Surgical revision required due to } \\
\text { recurrence of hernia. }\end{array}$} \\
\hline
\end{tabular}

patients is shown in Table 5. The overall healing rate was 17 out of $19(89.5 \%)$. With the exception of two patients with incomplete follow-up, all patients ultimately healed at a median time of 27 days (20-579 days). One patient served as an outlier and took 1.6 years ( 579 days) to heal because he was lost to follow-up until that time.

\section{DISCUSSION}

This small, retrospective series provides more data on the use of a modified CST combined with biologic mesh in the immunosuppressed transplant population with Chevron and Gibson incisions. This is an important paper since most of the literature that has been written on this topic consists of small patient series. We feel that a multidisciplinary approach is critical to 
Table 4. Patients with hernia recurrence

\begin{tabular}{|lcccc|}
\hline Patient & $\begin{array}{c}\text { No. of prior } \\
\text { VHR }\end{array}$ & $\begin{array}{c}\text { Time to } \\
\text { recurrence } \\
\text { (mon) }\end{array}$ & Intervention & Revision complications \\
\hline 1 & 4 & 25 & $\begin{array}{c}\text { Revision with external oblique rotational flap and Strattice } \\
\text { underlay }\end{array}$ & None, healing observed at POD 19 \\
2 & 0 & 8 & Revision with plication of abdominal wall and scar excision & $\begin{array}{c}\text { Developed hematoma on POD 16, drained on POD 22, } \\
\text { then lost to follow-up }\end{array}$ \\
3 & 53 & Abdominal binder & NA & \\
\hline VHR, ventral hernia repair; POD, postoperative day; NA, not applicable. &
\end{tabular}

Table 5. Complications of DM patients $(n=9)$

\begin{tabular}{|ll|}
\hline Patient & \multicolumn{1}{c|}{ Complications } \\
\hline No. of DM patients with complications (\%) & \multicolumn{1}{c|}{5 (55.6) } \\
Patient 1 & Seroma \\
Patient 2 & Seroma, hematoma, wound \\
& dehiscence \\
Patient 3 & Hernia recurrence, abdominal \\
& wall numbness \\
Patient 4 & Abscess \\
Patient 5 & Hernia recurrence \\
\hline DM, diabetes mellitus. & \\
\hline
\end{tabular}

achieve the best outcomes in this difficult patient population. Plastic surgery involvement in these cases is crucial for proper soft tissue management, closure techniques, and to perform the modified CST. The techniques are not always straightforward as a mid-line ventral hernia CST. Mesh placement, fascial approximation and dissection techniques should be modified as discussed previously.

The combination of these two techniques is supported by the literature. It has been argued whether synthetic or biologic mesh should be used in the transplant population with comorbidities on immunosuppression. It is known that biologic mesh has a decreased rate of infection compared to synthetic mesh, but an increased risk of hernia recurrence. Scheuerlein et al. [12] found an increased risk of recurrence with CST without mesh compared to a combination of CST with biological mesh. Findings of success with the combination of CST and biologic mesh have been demonstrated as compared to synthetic mesh in transplant patients. Brewer et al. [8] has demonstrated the advantage of biological mesh in transplant patients-the study found an $11.9 \%$ explantation rate of biologics in a series where $68 \%$ bridging mesh and 5\% component separation were utilized. In this same series, synthetic mesh resulted in a $69.2 \%$ explantation rate. Using synthetic mesh in this patient population has an increased risk of recurrence, infection, and further surgery. We do not feel the added complication of mesh infection outweighs the hernia recurrence rate. Our study showed that nearly all of our 19 pa- tients had procedures that resulted in successful reconstruction with the exception of three hernia recurrences (15.8\%). Of the patients experiencing recurrence, one patient was treated conservatively with an abdominal binder. The remaining two patients with recurrence were successfully repaired without complication. It is notable that none of the procedures required explantation of the mesh. Findings from Santangelo et al. [11] also show that biologic mesh was more successful than synthetic mesh in a 10-patient prospective case series because the biological mesh had a greater ability to integrate into the abdominal wall while minimizing risk of bacterial colonization and other complications. Although these studies suggest superiority of the biologic mesh, it has been noted that the porcine dermis collagen biologic mesh used in the 19 patients in this study is more rigid compared to synthetics, especially when using a large product size, making it more difficult to manage [11].

Because transplant patients are on immunosuppressants, they have an increased likelihood of fascial dehiscence, infections, and hernia recurrence [8]. Our data shows that only one of the 19 patients experienced wound dehiscence and one patient's procedure was complicated by an abscess. The relatively low infection rate $(5.3 \%)$ reported by our study is similar to other studies published such as Brewer et al. [8], in which a $15 \%$ incidence of infection was reported. This low infection rate may be due to the use of the biological mesh as compared to the synthetic mesh. When using a synthetic mesh any skin breakdown can lead to infection. However, biological mesh can be vascularized, allowing blood to flow into and through the area, bringing immune cells. A meta-analysis by Darehzereshki et al. [9] showed that the incidence of infectious wound complications was $10.9 \%$ with biologic mesh compared to $36.5 \%$ with nonbiologic mesh. Biological and synthetic mesh have equivocal recurrence rates [13]. Therefore, biological mesh remains the choice when having to choose between synthetic and biologic mesh due to its ability to be vascularized.

The data from our series also demonstrates acceptable longterm hernia recurrence rates of $10.0 \%$ and $22.2 \%$ after primary 
and recurrent hernia repairs respectively. Brewer et al. [8] reported a $24 \%$ hernia recurrence rate after $\mathrm{IH}$ repair in transplant patients, which is in line with our hernia recurrence rate. Li et al. [14] report a $22 \%$ hernia recurrence rate in renal transplant patients after $\mathrm{IH}$ repair with $\mathrm{CST}$ and tensor fascia lata grafts. Although a different combined procedure, the hernia recurrence rate is also similar to our patients.

Five of the nine patients with diabetes mellitus had complications in this series. Additionally, the only patient found to have a complication of infection (abscess) was diabetic. These results suggest a predisposition to complications in this subset of transplant patients. Diabetic patients are predisposed to poor glycemic control, increasing the risk of infection in the setting of surgery [15]. Also, wound healing is compromised in the diabetic patient due to glycosylation of vasculature leading to vasculopathy, immune cell dysfunction, and impaired proliferation and migration of fibroblasts $[16,17]$.

All patients with complete follow-up were found to be healed with a median healing time of 27 days. There are limitations to this conclusion. First, two patients did not have adequately documented follow-up due to limitations of our electronic medical record and one patient having follow-up at an outside institution. Therefore, our overall documented healing rate is $89.5 \%$. Furthermore, one patient experienced was not recorded to be healed until 1.59 postoperative years because they were lost to followup until this time.

Given the acceptable rates of healing and complications demonstrated in this case series, we believe that a modified open CST with biologic mesh is a safe and effective technique in the transplant population with abdominal hernias. The use of a multidisciplinary approach is critical to the success of the modified CST. Our study has several limitations of note including a small sample size of 19 . Furthermore, this study did not include any patients with active infection which is typically a contraindication to implantation of any prosthesis, including abdominal mesh, as only patients with a high likelihood of success would be selected to undergo this operation. Further studies on abdominal wall reconstruction in the transplant population are needed including prospective studies on the use of CST and biologic mesh to address the limits of our study.

\section{NOTES}

\section{Conflict of interest}

No potential conflict of interest relevant to this article was reported.

\section{Ethical approval}

The study was approved by the Institutional Review Board of Georgetown University Medical Center (IRB No. 2018-0008) and performed in accordance with the principles of the Declaration of Helsinki. Written informed consents were obtained.

\section{Patient consent}

The patients provided written informed consent for the publication and the use of their images.

\section{Author contribution}

Conceptualization: Sher SR, Evans KK. Data curation: Black CK, Zolper EG, Walters ET, Wang J, Martinez J, Tran A, Naz I, Kotha V. Writing - original draft: all authors. Writing - review \& editing: Black CK, Zolper EG, Sher SR, Evans KK. Approval of final manuscript: all authors.

\section{ORCID}

Cara K Black https://orcid.org/0000-0003-2137-1022 Elizabeth G Zolper https://orcid.org/0000-0001-6271-6918 Elliot T Walters https://orcid.org/0000-0002-8255-2858 Jessica Wang https://orcid.org/0000-0002-6242-5034 Jesus Martinez https://orcid.org/0000-0002-5150-0123 Andrew Tran https://orcid.org/0000-0001-9383-6605 Iram Naz https://orcid.org/0000-0001-8050-1822 Vikas Kotha https://orcid.org/0000-0003-1568-4284 Paul J Kim https://orcid.org/0000-0002-6186-6890 Sarah R Sher https://orcid.org/0000-0002-2419-2014 Karen K Evans https://orcid.org/0000-0003-1056-2114

\section{REFERENCES}

1. Smith CT, Katz MG, Foley D, et al. Incidence and risk factors of incisional hernia formation following abdominal organ transplantation. Surg Endosc 2015;29:398-404.

2. Humar A, Ramcharan T, Denny R, et al. Are wound complications after a kidney transplant more common with modern immunosuppression? Transplantation 2001;72:1920-3.

3. Vardanian AJ, Farmer DG, Ghobrial RM, et al. Incisional hernia after liver transplantation. J Am Coll Surg 2006;203: 421-5.

4. Kahn J, Muller H, Iberer F, et al. Incisional hernia following liver transplantation: incidence and predisposing factors. Clin Transplant 2007;21:423-6.

5. Ayvazoglu Soy EH, Kirnap M, Yildirim S, et al. Incisional hernia after liver transplant. Exp Clin Transplant 2017;15 (Suppl 1):185-9.

6. Ooms LS, Verhelst J, Jeekel J, et al. Incidence, risk factors, and 
treatment of incisional hernia after kidney transplantation: an analysis of 1,564 consecutive patients. Surgery 2016; 159:1407-11.

7. Piardi T, Audet M, Panaro F, et al. Incisional hernia repair after liver transplantation: role of the mesh. Transplant Proc 2010;42:1244-7.

8. Brewer MB, Rada EM, Milburn ML, et al. Human acellular dermal matrix for ventral hernia repair reduces morbidity in transplant patients. Hernia 2011;15:141-5.

9. Darehzereshki A, Goldfarb M, Zehetner J, et al. Biologic versus nonbiologic mesh in ventral hernia repair: a systematic review and meta-analysis. World J Surg 2014;38:40-50.

10. Coccolini F, Catena F, Bertuzzo VR, et al. Abdominal wall defect repair with biological prosthesis in transplanted patients: single center retrospective analysis and review of the literature. Updates Surg 2013;65:191-6.

11. Santangelo ML, Carlomagno N, Spiezia S, et al. Use of biological prostheses in transplant patients with incisional hernias: preliminary experience. Ann Ital Chir 2013;84:471-5.
12. Scheuerlein H, Thiessen A, Schug-Pass C, et al. What do we know about component separation techniques for abdominal wall hernia repair? Front Surg 2018;5:24.

13. Ibrahim AM, Vargas CR, Colakoglu S, et al. Properties of meshes used in hernia repair: a comprehensive review of synthetic and biologic meshes. J Reconstr Microsurg 2015; 31:83-94.

14. Li EN, Silverman RP, Goldberg NH. Incisional hernia repair in renal transplantation patients. Hernia 2005;9:231-7.

15. Kwon S, Thompson R, Dellinger P, et al. Importance of perioperative glycemic control in general surgery: a report from the Surgical Care and Outcomes Assessment Program. Ann Surg 2013;257:8-14.

16. Buranasin P, Mizutani K, Iwasaki K, et al. High glucose-induced oxidative stress impairs proliferation and migration of human gingival fibroblasts. PLoS One 2018;13:e0201855.

17. Wong SL, Demers M, Martinod K, et al. Diabetes primes neutrophils to undergo NETosis, which impairs wound healing. Nat Med 2015;21:815-9. 